\title{
Efectividad de la gestión en las universidades, desde modelos de percepción de calidad de estudiantes: el modelo de la Universidad Bernardo O 'Higgins
}

\author{
RufF ESCOBAR, Claudio \\ Universidad Bernardo O'Higgins (Santiago de Chile)* \\ Correo electrónico: cruff@ubo.cl \\ RUIZ TOLEDO, MARCELO* \\ Correo electrónico: mruiz@ubo.cl \\ MATHEu PÉREZ, AleXIS* \\ Correo electrónico: alexis.matheu@ubo.cl \\ JUICA MARTÍNEZ, PAOLA* \\ Correo electrónico: paola.juica@ubo.cl \\ ANABALÓN ARENAS, GERARDO* \\ Correo electrónico: gerardo.anabalon@ubo.cl
}

\begin{abstract}
RESUMEN
La calidad en la educación es un tema recurrente en esta sociedad donde la formación de profesionales competentes es un elemento esencial frente a las crecientes demandas del mercado. La competitividad, dada por la multivariedad de ofertas educativas, junto con el rol histórico que tienen las universidades como gestoras de conocimiento, impulsa a las universidades a centrarse en un proceso permanente de reflexión y análisis acerca del cumplimiento de sus objetivos institucionales y del grado de efectividad medido en la satisfacción de sus principales clientes, es decir, sus estudiantes. En este contexto, la presente investigación tiene como objetivo otorgar información para establecer prioridades de gestión financiera e institucional desde el grado de satisfacción de los estudiantes de la Universidad Bernardo O’Higgins aplicando la metodología de modelación de ecuaciones factoriales, buscando relaciones entre diferentes áreas de satisfacción, con métodos multivariados como el análisis factorial. Se ha empleado el método de consistencia interna basado en la medida KMO y la prueba de esfericidad de Bartlett. Finalmente, el estudio ha logrado modelar un sistema derivado del comportamiento de respuestas de estudiantes y docentes.
\end{abstract}

Palabras clave: calidad, efectividad, evaluación, educación superior, cultura de servicio, encuesta de satisfacción.

Clasificación JEL: I23; C02.

MSC2010: 62; 62P20.

Artículo recibido el 24 de septiembre de 2019 y aceptado el 26 de enero de 2020 


\title{
Effectiveness of management in the universities, from models of perception of student quality: the model of the University Bernardo O'Higgins
}

\begin{abstract}
Quality in education is a recurring theme in this society where the training of competent professionals is an essential element for their progress and development in the face of growing market demands. The competitiveness given by the multivariety of educational offerings, together with the historical role of universities as knowledge managers, drives universities to focus on a permanent process of reflection and analysis about the fulfillment of their institutional objectives and the degree of effectiveness measured in the satisfaction of their main clients, that is, their students. In this context, this research aims to establish priorities for financial and institutional management from the degree of satisfaction of university students at Bernardo O'Higgins University by applying the methodology of modeling structural equations, based on variances, seeking relationships between different areas of satisfaction, with multivariate methods factorial analysis. For these purposes, the internal consistency method based is given by the average of the correlations between the items in order to estimate the reliability of his instrument. Finally, it should be pointed out that the study has managed to model a system derived from the response behaviour of students and teachers at the Universidad Bernardo O 'Higgins.
\end{abstract}

Keywords: quality, effectiveness, evaluation, higher education, service culture, satisfaction survey.

JEL classification: I23; C02.

MSC2010: 62; 62P20.

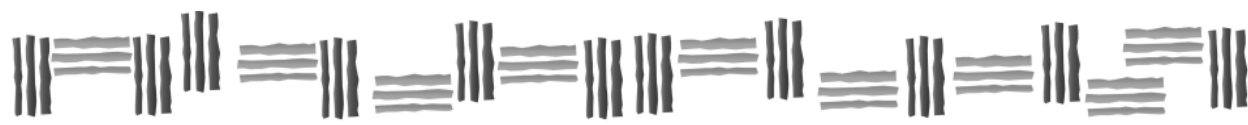




\section{Introducción.}

Si se reflexiona acerca de la esencia del paradigma actual, entre los conceptos que desfilan como connaturales de esta sociedad se destacan el valor del derecho y el requerimiento de calidad en los servicios. Este hecho se traduce en que hoy, más que antaño, existe una mayor conciencia de lo que es demandable y exigible a nivel social.

El sistema educativo no ha estado exento de estos requerimientos, ya que es constantemente interpelado a evidenciar su desempeño en el marco de los estándares de calidad, no solo en lo que alude al cumplimiento de sus macroobjetivos institucionales, sino en la efectividad de todos y cada uno de sus servicios. El desafío radica entonces en poder generar a nivel institucional, mecanismos de medición de la calidad de sus servicios capaces de recopilar y analizar información, suficiente y fidedigna de la realidad institucional, en todos sus niveles y desde este conocimiento, sustentar las políticas y las estrategias de acción necesaria en favor de la efectividad de sus servicios (Cabrera, 2005; Lepeley, 2001).

En este nuevo escenario social, las universidades son, como han sido siempre, hitos culturales para las naciones (Salmi, 2009). Es así como, han asumido como parte de sus funciones, la promoción de la calidad, ya que se espera que ellas sean garantes en la construcción de sociedades de conocimiento, comprometidas, a nivel global y local, en potenciar el desarrollo cultural, político, social y económico de las naciones. De esta manera, el crear universidades eficientes y competitivas, impulsadas por el conocimiento, ayuda a los países a su crecimiento, ya que, "la aplicación del conocimiento da lugar a formas más eficientes de producir bienes y servicios, y de suministrarlos de una manera más eficaz, a un menor costo y a un mayor número de personas" (Salmi, 2009, p. 16).

Una de las maneras que han encontrado los organismos de educación, especialmente, las universidades, para medir la calidad de sus servicios son las denominadas "encuestas de satisfacción" donde sus propios estudiantes son interpelados a manifestarse con respecto a una serie de temas importantes acerca del sistema de formación, aspectos de gestión, administrativos, servicios básicos, entre otros (Blanco-Guillermety \& Blanco-Peck, 2018; Beltyukova \& Fox, 2002).

Este mecanismo de medición de calidad sitúa como base fundamental la interacción humana y la opinión de las personas, ya que genera los espacios libres de participación, con el propósito de conocer y entender la percepción de los mismos estudiantes acerca de los procesos que viven. Esto humaniza y democratiza el sistema ya que se aquilata la valoración de los propios protagonistas del proceso de formación. Cabe destacar que este hecho, en el caso de la educación, complementa y trasciende los términos empresariales de "proveer servicios" para la "satisfacción del cliente", entendiendo que éste, es decir, el estudiante, forma parte y es actor de su propio desarrollo y el de la institución que ha elegido y a la que se ha adscrito (Gaitán \& López, 1999). En otras palabras, "la medición del grado de satisfacción de los estudiantes clientes es uno de los indicadores utilizados para medir calidad de servicios, retener a los alumnos y desarrollar un ambiente democrático" (Blanco-Guillermety \& BlancoPeck, 2018, p. 128).

Junto con el incremento explosivo de la demanda por calidad en la educación, se advierte el hecho de que, en consecuencia con lo antes descrito, la educación ha debido ingresar al "mercado competitivo" lo que la obliga a medirse con otros competidores donde el prestigio y la tradición desempeña un rol preponderante (Arambewela \& Hall, 2006).

Según estas nuevas exigencias, el constituirse como una fuerza competitiva dentro del mercado requiere de las universidades la generación de un ambiente orientado al servicio, que motive a la propia institución a una investigación exhaustiva, acompañada de una profunda reflexión acerca de sus propios procesos, a saber:

Las universidades que aspiran a mejores resultados se comprometen a evaluar de una manera objetiva sus fortalezas y las áreas que necesitan mejorar, a establecer nuevos objetivos, y a diseñar 
y poner en práctica un plan de renovación que pueda conducir a un mejor desempeño de su misión. (Salmi, 2009, p. 67)

Lo descrito implica cultivar una "cultura de servicio" que considere aspectos importantes como, asegurar que la variedad de los programas educativos que ofrece al mercado responde a las demandas sociales y que certifique que sus profesionales egresados y titulados cuenten con las competencias declaradas en el perfil de sus carreras; asimismo, que puedan ser empleados y logren un buen desempeño en el ejercicio de sus profesiones. De esta manera, este cambio de enfoque que promueve una mirada de la educación como un servicio hace pertinente la evaluación de las necesidades de sus clientes-estudiantes en vínculo con las necesidades sociales del mundo laboral (Chung \& McLarney, 2000).

Es significativo destacar que este nuevo enfoque invita a las universidades a una transformación estratégica e innovadora y a persistir en sus esfuerzos por ser eficientes y oportunas en responder a las necesidades de sus estudiantes (Salmi, 2009).

Un factor sustancial a considerar en todo momento es que estas transformaciones no pueden responder a fórmulas de acciones estandarizadas ya que cada institución debe reconocer a la diversidad de sus estudiantes y actuar según su propio modelo de educativo, bajo una continua evaluación que oriente sus procesos.

\section{Calidad y educación.}

Desde su génesis el concepto de calidad está relacionado con la oferta de productos; sin embargo, hoy en día este término es aplicable a muchas áreas, incluyendo la educación. Por lo señalado se concibe que medir la calidad de un producto es tanto más fácil que medir la calidad de un servicio, entendiendo esta significación como cualquier acción para satisfacer las necesidades un cliente (Vázquez et al., 2017). Por esta razón, el consensuar qué se comprende por calidad en los servicios es una labor difícil, más aún cuando se enmarca en el ámbito de la educación superior, ya que la calidad en los servicios educativos es un constructo de naturaleza multidimensional, un valor intangible y abstracto (Mejías et al., 2013). Además, se basa en elementos que diferencian al servicio tales como; su intangibilidad, su diversidad, inseparabilidad y caducidad (Parasuraman et al., 1985).

A modo de consenso, en educación se entiende por calidad de servicio como "aquellas acciones o actividades que se reflejan en la conformidad y la satisfacción que experimenta la comunidad académica sobre los diferentes servicios prestados por ésta" (Vázquez et al., 2017, p. 74).

Por otro lado, Parasuraman et al. (1985) señalan que la calidad de servicio es entendida como la discrepancia entre lo que el cliente percibe como "calidad del servicio" y las "expectativas" que tenía de él. Esta definición tiene un enfoque perceptivo (Grönroos, 1994) que avala el sistema de encuestas de satisfacción o "SERVQUAL" (Parasuraman et al., 1985; Salvador, 2010).

Existen diferentes puntos de vista sobre la valoración que se puede realizar sobre las encuestas de satisfacción como instrumentos para medir calidad. Las principales críticas a esta herramienta de evaluación apuntan justamente a su subjetividad dada por su enfoque perceptivo. Sin embargo, otros autores como Shekarchizadeh et al., (2011) y Mejías et al. (2013) aseguran que este método es eficaz en tanto que evidencia información de calidad.

Algunos elementos que señala la literatura como indicadores o variables de satisfacción estudiantil son: sentido de pertenencia e identificación con la institución, compromiso y preocupación por los estudiantes, calidad de los programas, ayudas económicas, apoyo académico, efectividad en el proceso de matrícula, seguridad, actividades sociales y de integración, ambiente universitario, labor 
académica y administrativa, servicios computacionales, casino, biblioteca, entre otros (BlancoGuillermety \& Blanco-Peck, 2018).

De esta manera, el concepto de calidad en educación superior se puede traducir a indicadores que son propuestos por diferentes autores como percepciones de calidad de servicio. Entre ellos, los que guardan relación con aspectos académicos como la calidad en la enseñanza y competitividad de los docentes, su actitud y vínculo con los alumnos, metodologías de aula (Capelleras \& Veciana, 2004; LeBlanc \& Nguyen, 1997; Camisón, Gil \& Roca, 1999). Otros que se relacionan con el currículum, los programas de estudio, los recursos académicos, la titulación oportuna (LeBlanc \& Nguyen, 1997; Owlia \& Aspinwall, 1996). Finalmente, los de índole administrativo, de equipamiento, localización, seguridad, servicios de asesoramiento, actividades sociales, entre otros (Kwan \& Ng, 1999; Joseph \& Joseph, 1997; Bigné et al., 1997).

\subsection{Modelos de evaluación de calidad.}

En términos de evidencias, el proceso de acreditación se yergue como garantía de calidad ya que ella ha dado fin a la parcialización y aislamiento de las universidades en términos de evaluación de sus procesos y ha unificado y externalizado los criterios y la labor de medición, más aún en el caso de países como Chile cuya financiación está supeditada a los mecanismos de acreditación (De la Fuente et al., 2010).

Entre los modelos de la evaluación de calidad se distinguen aquellos que enfatizan y priorizan los programas académicos por la estructura y los servicios administrativos; otros, enmarcados en los procesos de acreditación y rendición de cuentas, frente a los modelos sistémicos que consideran a las universidades dentro del sistema social. Por otra parte, el modelo global pone atención a los resultados obtenidos en relación con los objetivos institucionales y el modelo etnográfico construye una descripción científica de los procesos educacionales (Morresi et al., 2008). Ante estas propuestas que ofrece la literatura como aplicables para medir la calidad de una institución de educación superior, se destacan algunos elementos de análisis comunes, tales como el valor del recurso humano, medido en el nivel de independencia y autonomía que tienen sus profesores para ejercer la labor docente, lo que, según Mintzberg et al. (1991) constituye un riesgo porque no existe ningún control de lo que en términos medibles el docente decide hacer en favor de la formación de sus alumnos y del desarrollo de la institución, grado de adaptabilidad a los cambios, flexibilidad para enfrentar los nuevos desafíos acogiendo la innovación y los avances en la tecnología.

Los modelos de docencia, centrados en el estudiante y avalados en una estructura organizativa que permita la modificación de los roles, estimulando el protagonismo de los educandos, a través del control del ejercicio pedagógico en miras de la autonomía y autorregulación.

Una evaluación de rendimiento que acrediten la calidad de la enseñanza determinada por la calidad docente (Blanco-Guillermety, \& Blanco-Peck, 2018).

Basándose en lo señalado se puede abordar el tema de la calidad en educación superior desde variados aspectos, como por ejemplo, en función a la calidad entendida como prestigio institucional, desde la disponibilidad de recursos humanos y materiales, como un concepto relacionado con la efectividad manifiesta en resultados (matrícula, tasa de aprobación, titulación oportuna, empleabilidad etc.) o entender la calidad como un valor diferenciador y gestor de cambio explicitado en el desarrollo social y académico de sus alumnos, entre otros (Morresi et al., 2008; Hermida et al., 2008).

\subsection{Calidad docente, evaluación de rendimiento y valoración estudiantil.}

La evaluación de rendimiento que mide la calidad y la efectividad de la labor docente es un proceso planificado, intencionado, sistemático y continuo que repercute, directamente, en los índices de retención estudiantil. Además, se transforma en una herramienta descriptiva que resguarda el cumplimiento del modelo pedagógico institucional. Este elemento se mide de manera directa, a través 
de las encuestas de satisfacción estudiantil, ya que son los estudiantes los que se vinculan directamente con la actividad pedagógica de sus profesores.

Un factor que debe ser considerado en este punto de análisis es el comprender la evaluación de rendimiento no como un valor meramente instrumental, como si se tratara solo de elementos de análisis segmentado. Por el contrario, el valor de esta medición radica en su perfil sistémico y flexible, adecuado a los objetivos propios de cada institución, y que garantiza integridad y objetividad ya que es un sistema que debe incorporar diferentes fuentes de información, en diferentes etapas del proceso (BlancoGuillermety, \& Blanco-Peck, 2018).

Se suma a lo anteriormente enunciado, el hecho que la evaluación de rendimiento tiene un perfil participativo en la medida que se adhiere a la evaluación de los estudiantes, la de los docentes que se hacen parte de ella en la etapa de autoevaluación. Este sistema de evaluación aporta dinamismo a las relaciones institucionales, ya que éstas, al tomar las consideraciones de dicha evaluación, son interpeladas a rediseñarse continuamente.

Claro está que este proceso de evaluación y mejora continua no solo tiene que considerar como parte del proceso de formación de un alumno lo que sucede en el aula, sino más bien, debe tener una mirada holística e integral de las condiciones de desarrollo de los estudiantes, considerando que:

Para algunos estudiantes, el proceso de estudio no solamente representa la adquisición de ciertas destrezas y conocimiento teórico, sino que está muy relacionado a su desarrollo social y a su crecimiento personal. Por estas razones, su satisfacción en relación con la institución es muy importante, aunque muchas instituciones han olvidado tomar en consideración y luego se quejan porque su retención es baja. (BlancoGuillermety, \& Blanco-Peck, 2018, p. 129)

\subsection{Educación, valor de mercado y satisfacción del cliente.}

En este nuevo paradigma, el considerar a la educación dentro del valor mercantil propio de cualquier empresa suscita una gran controversia y posiciones encontradas. Sin embargo, ambas se centran en los alumnos, las demandas sociales, las empresas como principales grupos de interés. Bajo esta mirada y con el propósito de abarcar las múltiples dimensiones y tareas que asumen las universidades y en consecuencia ampliando la idea de lo que se define como "cliente" en educación superior, De la Fuente et al. (2010) señala que:

La literatura especializada propone diferentes grupos de clientes: estudiantes, empleadores, organizaciones que contratan a los graduados, personal docente e investigador, sociedad, administración pública, familias, personal administrativo y de servicios, donantes y/o patrocinadores y otros grupos (proveedores, futuros estudiantes, etc.), si bien cada uno de estos grupos recibe fruto de uno de los diversos procesos llevados a cabo por la universidad. (p. 351)

La satisfacción es un concepto que alude a la valoración positiva frente a un servicio (Oliver, 1980) es un juicio personal, emotivo y cognitivo que relaciona causas y consecuencias y que influye directamente en el actuar y en las tendencias del consumidor (Cronin, et al, 2000). Por lo tanto, son agentes predictores de comportamiento (Westbrook, 1987).

En referencia a todo lo señalado, se puede determinar que la calidad de servicios en educación superior es un concepto complejo, considerando las funciones y la finalidad del sistema educativo (Sverdlick, 2012). Por lo tanto, es contextual, atribuible a una multidimensionalidad de elementos, dinámicos y evolutivos que conforman el modelo educativo de una institución y que se ciñe a estándares establecidos de un modelo de gestión de calidad consensuado y explicitado en estándares de acreditación institucional.

Ante el reto de los mercados en su expresión económica, política y social, las instituciones de educación superior deben ser capaces de asegurar que sus profesionales egresados puedan acceder de manera efectiva a la labor productiva y esto se cumple, en mayor medida, cuando las instituciones de 
educación trabajan de manera innovadora y flexible en intercambio con el entorno productivo, de modo que:

La educación superior es un proyecto social que no termina en la educación y la formación profesional. Necesita que los egresados, efectivamente, aseguren una oportunidad para desempeñar productivamente su educación. Discutir esto sugiere que la intermediación que en el mercado profesional realiza la educación superior, si bien es positiva en términos de la oportunidad de acceso a la educación, tiene problemas en la forma como se organiza el proceso educativo para la transición al mundo del trabajo. (Lagarda, 2001, p. 7)

Uno de los puntos dominantes al analizar la calidad de la educación y su efectividad de los servicios es la llamada "pertinencia", es decir, la relevancia a dar respuesta, desde los sistemas de formación superior, a las demandas del mercado y del sector productivo (Tünnermann, 2006). Pero esto no significa que las universidades se deban limitar en una acción coartada de respuesta a las demandas, sino más bien, deben considerar, de una manera más amplia, los requerimientos globales que la sociedad pide de las instituciones de educación superior. Entendiendo que:

La pertinencia tiene que ver con el "deber ser" de las Universidades, es decir, con una imagen deseable de las mismas. Un "deber ser", por cierto, ligado a los grandes objetivos, necesidades y carencias de la sociedad en que ellas están insertas y a los retos del nuevo contexto mundial. La preocupación por la pertinencia de la educación superior en la sociedad. (Tunnermann, 2006, p.1)

De esta manera, la preocupación por la pertinencia, directamente relacionada con la calidad de los servicios, insta a la educación superior a ser creativas, a traducir sus metas en aspectos medibles que aseguren el cumplimiento de sus objetivos en función de una sociedad contemporánea cada vez más exigente.

\subsection{La Universidad Bernardo O’Higgins y su política de aseguramiento de la calidad.}

La Universidad Bernardo O'Higgins, como una institución comprometida con la formación de sus estudiantes y con el desarrollo de la sociedad, ha creado un sistema de aseguramiento de la calidad capaz de velar por el cumplimiento de los objetivos y metas institucionales. En consecuencia, ha declarado como meta principal el poder lograr que sus estudiantes alcancen, de manera efectiva y exitosa, el término oportuno de su formación universitaria con la adquisición de su título profesional, cumpliendo con todos los compromisos formativos declarados en el perfil de egresado. Todo lo anterior, resguardado bajo el sello propio de la institución, caracterizado por la formación integral y los valores de compromiso social, el valor al mérito, el espíritu de servicio, el énfasis en el aprendizaje y la pertinente actualización de los desafíos disciplinares y profesionales.

El "Sistema de Aseguramiento de la Calidad de la Universidad Bernardo O'Higgins (SAC UBO)" vincula y articula los principales componentes y áreas del sistema institucional, gestionando de manera ordenada, sistémica, planificada y controlada el cumplimiento de la misión y de la visión institucional. Basándose en este objetivo determina cuáles son los procesos que deben ser monitoreados y qué tipo de información es relevante para la evaluación de los mismos. Además, sigue a esta etapa de control, análisis y contrastación de los indicadores evaluados contra los propósitos y objetivos, el diseñar remedios y políticas de mejora que den respuesta a los planes de mejora continua.

\section{Metodología.}

\subsection{Antecedentes.}

La calidad en el servicio es un concepto que se ha definido y discutido de variadas maneras y además la forma de ser evaluado es un tema muy complejo y debe ser visto desde varios niveles. Este indicador de calidad es requisito de competitividad (Grönroos, 1994), siendo las claves del éxito, no solo su contextualización sino, además, el aseguramiento en el tiempo y la satisfacción de los usuarios en el contexto de la eficacia. 
Como se ha visto en la revisión bibliográfica, el concepto ha transitado por variadas definiciones de diversos autores. En este estudio se entiende la calidad de los servicios, desde la percepción que tienen los estudiantes sobre cómo se han visto satisfechas sus necesidades. Esta investigación es trasversal de tipo censal (Sampier, Fernández \& Baptista, 2010), con análisis estadístico de datos de todas las carreras en los períodos 2017 y 2018 en la Universidad Bernardo O’Higgins.

\subsection{Métodos de análisis.}

La investigación se basa en la metodología de modelación de ecuaciones factoriales, basados en las varianzas, buscando relaciones entre diferentes áreas de satisfacción. Estos modelos estadísticos permiten estimar el efecto y las relaciones entre múltiples variables, es decir, modelos de análisis factorial que permiten efectos directos e indirectos entre los factores creados, a través de las correlaciones existentes entre las variables medidas. Este método es la unión de dos tradiciones (Chin, 1998), a saber, la teoría econométrica que se enfoca en la predicción y el enfoque psicométrico que modela conceptos como variables latentes (no observadas) que son indirectamente inferidas de múltiples medidas observadas (indicadores o variables manifiestas).

Lo descrito corresponde a una técnica que combina tanto la regresión múltiple como el análisis factorial, dicho de otro modo, permite, no solo, evaluar las complejas interrelaciones de dependencia, sino también incorporar los efectos de los errores de las medidas.

\subsubsection{Análisis estadístico del método}

Este método se basa en las etapas que a continuación se describen:

a) Plantear estructura de constructos-ítems a priori: medición de variables "latentes" no observables directamente.

b) Construir un diagrama del modelo y medida.

c) Seleccionar la matriz de datos (input): pueden ser usadas matriz de varianza-covarianza o matriz de correlaciones, además de relaciones entre variables observadas.

d) Estimar el modelo: de existir normalidad, se usará el método de estimación máximo verosímil (ML) por ser eficiente y no sesgado; de no existir normalidad, podrían usarse los métodos de Mínimos Cuadrados Ponderados (WLS), Mínimos Cuadrados Generalizados (GLS) y Asintóticamente Libre de Distribución (AGL).

e) Evaluar la bondad de ajuste al modelo de análisis factorial mediante las medidas KMO y esfericidad de Bartlett.

f) Interpretar y modificar el modelo (si está justificado). Serán analizados los siguientes aspectos; asociación entre ítems y constructos, nivel de explicación de las variables observadas, validación de escalas de medida (validez y fiabilidad), validez convergente (grado en el que las medidas utilizadas para medir un concepto latente están relacionadas entre sí), validez discriminante (grado en el que dos conceptos latentes especificados son diferentes, aunque estén correlacionados entre sí). Todo lo descrito se entregará a través de los siguientes elementos:

- Matriz de correlaciones entre todas las variables (constructos) incluidas en el modelo de investigación.

- Media y desviación típica de los factores / constructos.

- Índices de bondad de ajuste.

- Parámetros estimados: $\lambda$ (validez convergente).

- Intervalos de confianza entre pares de factores (v. discriminante)

- Coeficientes de fiabilidad.

- Coeficiente Kaiser-Meyer-Olkin (KMO).

- Test de esfericidad de Bartlett.

- Coeficiente de fiabilidad compuesto.

- Análisis de la Varianza Extraída (AVE). 


\subsection{Encuesta de satisfacción.}

La Universidad Bernardo O'Higgins se ha destacado en el tiempo por entregar un servicio de calidad, considerando las necesidades de sus alumnos. Es por esta razón, que es imprescindible conocer la opinión de estos, sus percepciones y su nivel de satisfacción con el servicio recibido por la Universidad en sus diferentes puntos de contacto.

Bajo este contexto, IPSOS Chile ha desarrollado un estudio que permite conocer esta información y visualizarla, desde lo más general a lo específico, con respecto del servicio entregado.

La encuesta de satisfacción es realizada a través del grado de satisfacción medido en una escala del 1 al 7 (cumple con las condiciones de una escala Likert), la forma de entrega de resultados se detalla en la Figura 1. La encuesta es de alta confiabilidad estadística, y es de tipo censal.

Figura 1. Forma de entregar los resultados de la encuesta.

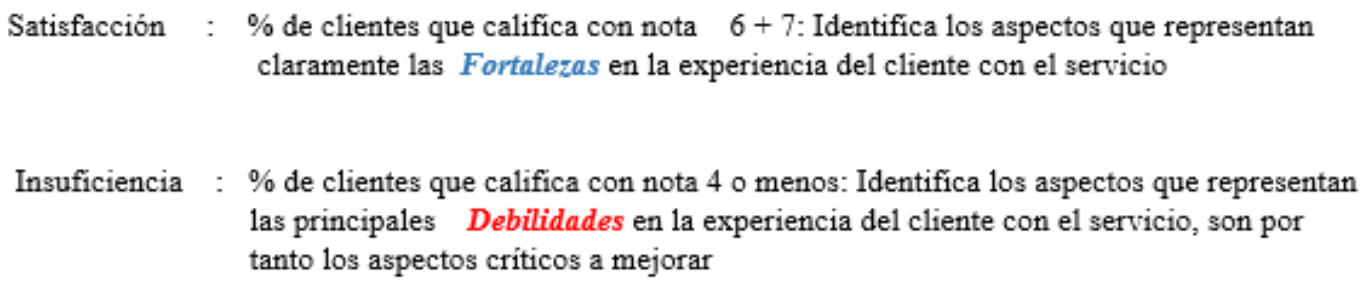

Fuente: Elaboración propia.

La Figura 2 muestra cómo se entiende la lealtad a la institución.

Figura 2. Forma de cálculo de alumnos leales a la UBO.

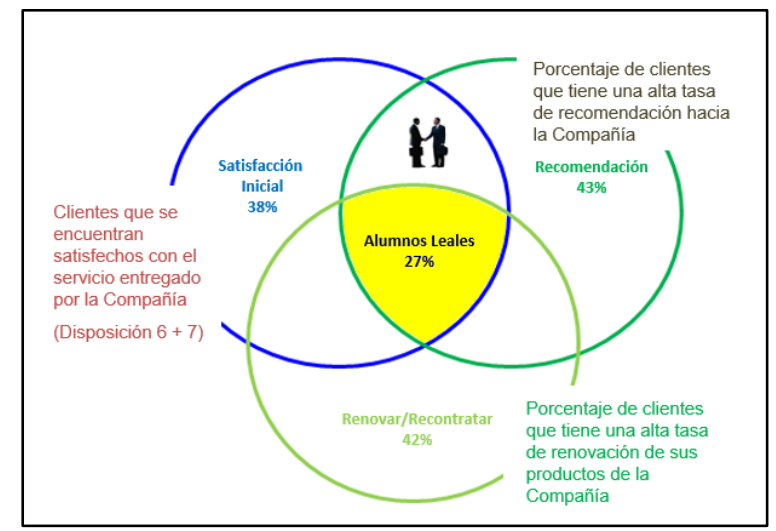

Fuente: Elaboración propia.

\section{Resultados de creación del modelo teórico UBO.}

El fenómeno de los servicios universitarios y sus relaciones con la calidad de la educación terciaria, como se ha visto, es un fenómeno complejo; por eso las ecuaciones factoriales pueden dar respuestas a las interrelaciones entre variables múltiples (variables que pueden cumplir a la vez ser independientes 
o independientes). El modelo UBO (Universidad Bernardo O'Higgins, 2018), por lo tanto, busca entender interrelaciones de causalidad, desde la contrastación de la teoría factorial de satisfacción general, partiendo de la satisfacción de los servicios, en un modelo de contrataste de hipótesis.

El sistema, por tanto, buscará la estimación simultánea de relaciones de dependencia múltiples e interrelacionadas y la capacidad para representar conceptos no observables (actitudes, percepciones, intenciones) en las relaciones, basado en la representación gráfica de las relaciones de causalidad.

\subsection{Definición y conceptos básicos.}

El sistema consta de dos componentes, el primero es el modelo factorial derivado del diagrama que representa las relacionales causales y el segundo modelo de medida o factorial dado las relaciones entre indicadores (variables observadas) y constructos (variables no observadas) (Diagrama 1 y 2 ).

Diagrama 1. Modelo Factorial UBO.

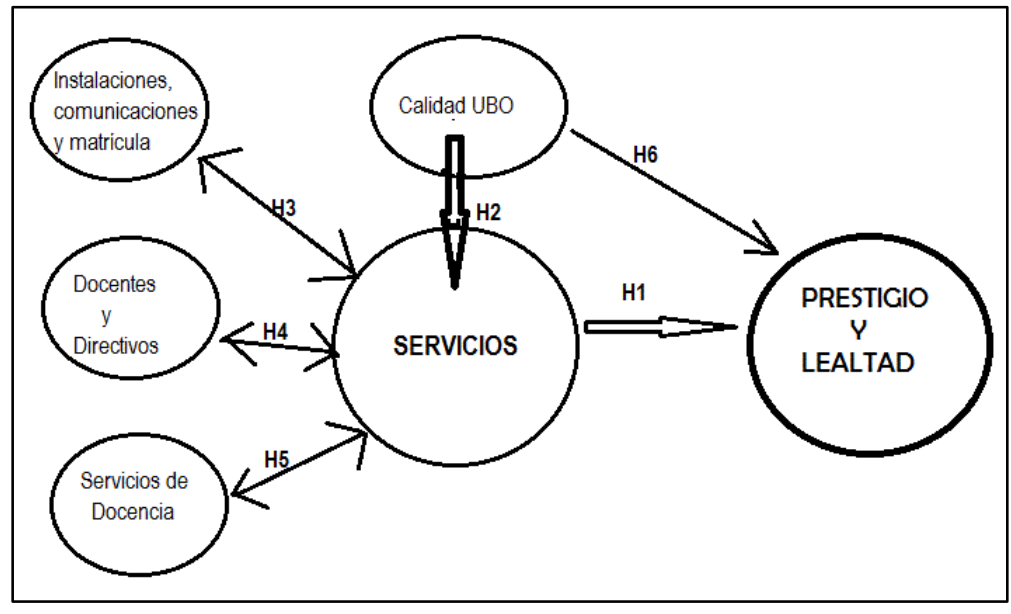

Fuente: Elaboración propia.

En el Diagrama 1 las relaciones están dadas por:

$$
\begin{gathered}
P y L=\beta 1 * C+\beta 2 * S+E 1 \\
\mathrm{~S}=\beta 3 * C+\beta 4 * I C M+\beta 5 * D D+\beta 6 * S D+E 2 \\
\mathrm{ICM}=\beta 7 * S+E 3 \\
\mathrm{DD}=\beta 8 * S+E 4 \\
\mathrm{SD}=\beta 9 * S+E 5
\end{gathered}
$$

donde $\beta$ son los coeficientes efectos variables explicativa / variables explicada y $E$ el efecto aleatorio.

Varios autores como Zineldin et al. (2011), han planteado que la satisfacción es un espejo del rendimiento del servicio, por lo que la intención de prestigio y lealtad debe estar directamente relacionada con el servicio, por lo que del modelo sugerido nacen varias hipótesis: 
H1. El servicio tiene una influencia directa, positiva y significativa sobre el prestigio y lealtad.

H2. La calidad UBO tiene una influencia directa, positiva y significativa sobre el servicio.

$\mathbf{H 3 , 4 , 5}$. El servicio tiene una influencia directa, positiva y significativa sobre el servicio de docencia, docentes y directivos, así como, en la infraestructura comunicaciones y procesos de matrícula.

H6. La calidad UBO tiene una influencia directa, positiva y significativa sobre el prestigio y lealtad.

\section{Diagrama 2. Modelo de Medida UBO.}

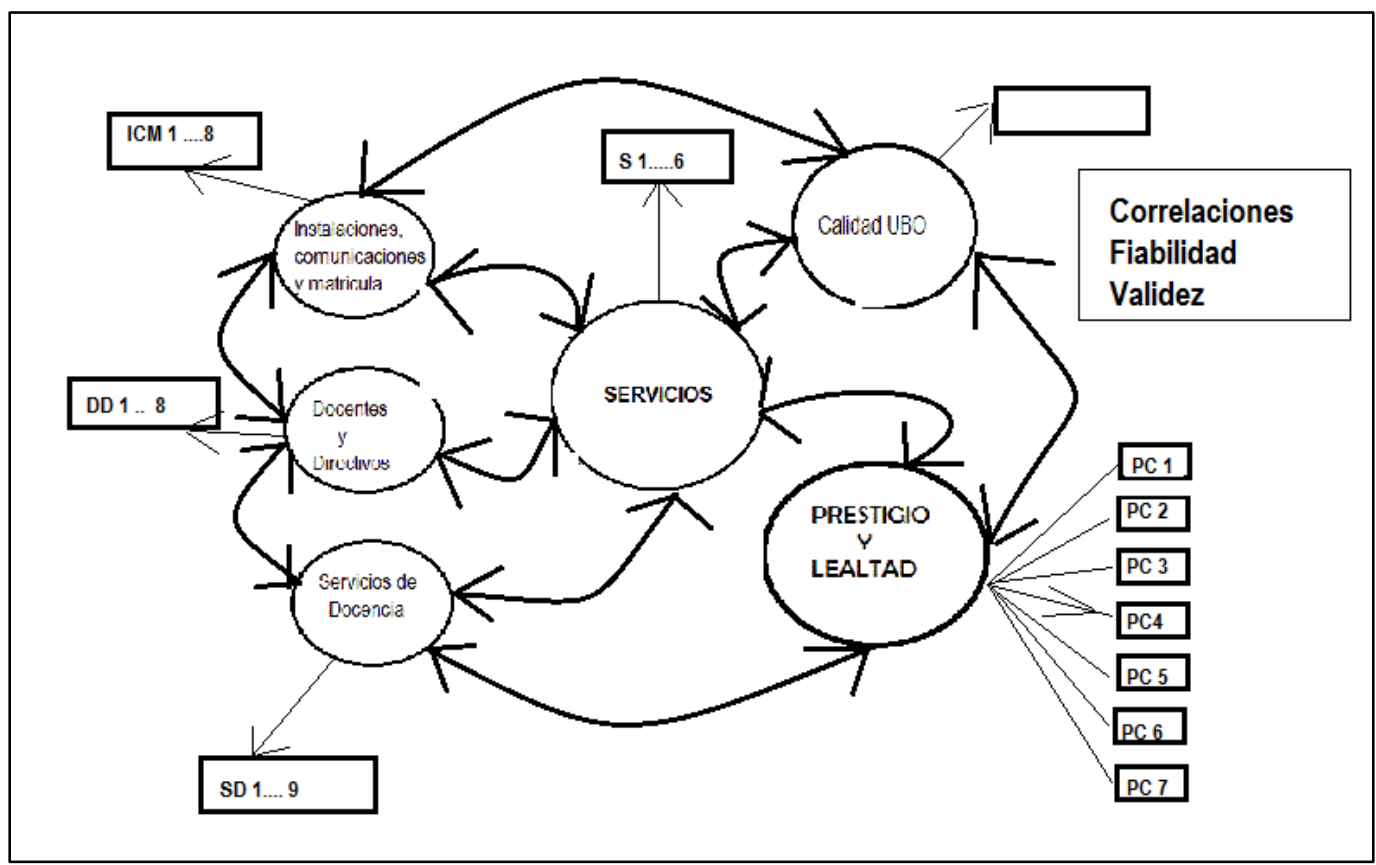

Fuente: Elaboración propia.

Las ventajas de este modelo UBO son:

1) Mayor flexibilidad estadística (permite interpretación incluso en presencia de multicolinealidad).

2) Uso del Análisis Factorial C.

3) Confirmatorio para reducir el error de medida (múltiples indicadores por variable latente).

4) Posibilidad de contrastar modelos conjuntamente.

5) Interfaz de representación gráfica.

6) Permite:

- Contrastar modelos con múltiples variables dependientes.

- Incorporar variables mediadoras.

- Modelizar y medir los errores.

- Comparar el modelo para submuestras diferentes (multimuestra).

- Utilizar datos difíciles (distribución no normal, datos ausentes).

\subsection{Resultados básicos preliminares de la encuesta de satisfacción 2017-2018.}

Los principales resultados descriptivos en el período 2017-2018, parten con las representaciones de los estudiantes leales UBO, que ha transitado como se observa en el Gráfico 1 de un $37 \%$ a un $34 \%$, en este leve retroceso. También se observan disminuciones en los tres indicadores que conforman la variable: renovación, recomendación y satisfacción inicial. 
Gráfico 1. Alumnos Leales UBO 2017 y 2018.

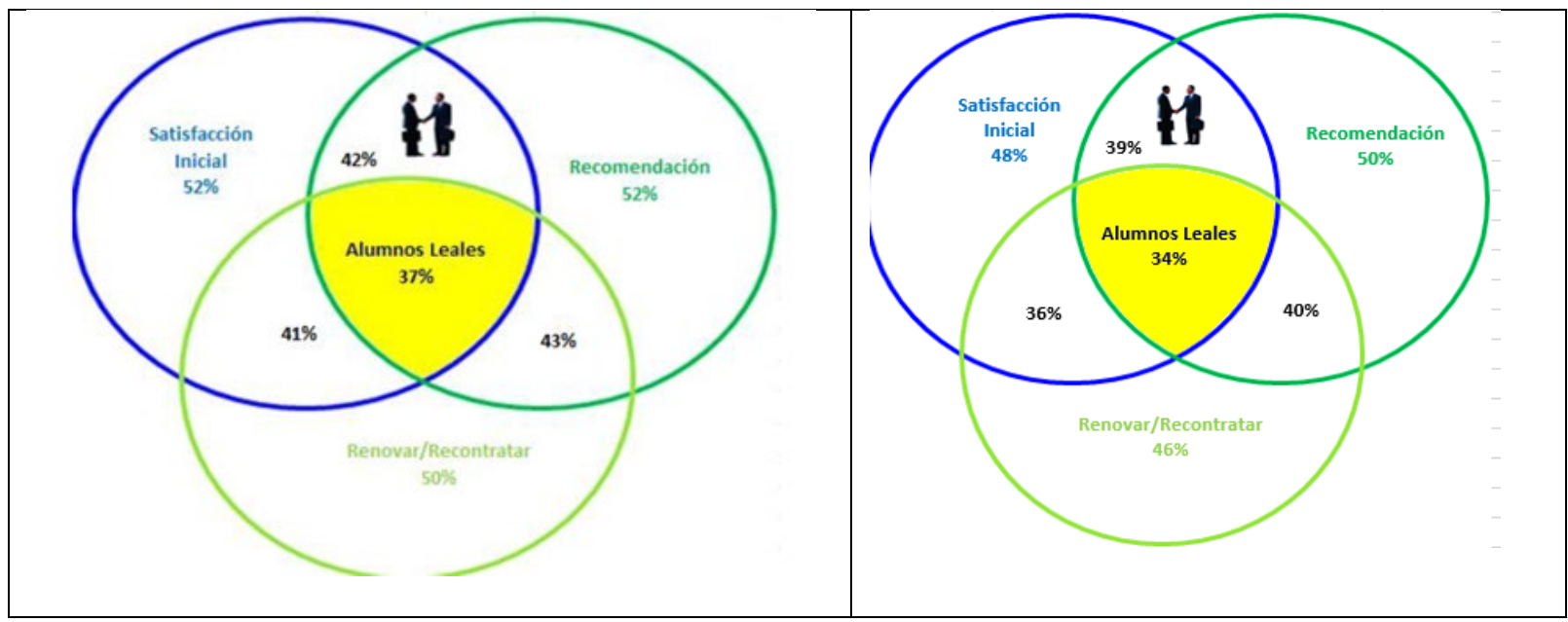

Fuente: Elaboración propia.

Las tablas siguientes detallan los resultados de todas las variables no observables (constructos) y los indicadores (variables observables) de estas variables.

La Tabla 1 muestra los resultados de la variable Prestigio y Lealtad que es más bajo en la percepción de los estudiantes de la Facultad de Salud.

Tabla 1. Variable Prestigio y Lealtad UBO 2017 y 2018.

\begin{tabular}{|c|c|c|c|c|c|c|c|}
\hline Preguntas & $\begin{array}{l}\text { UBO } \\
2017\end{array}$ & $\begin{array}{l}\text { UBO } \\
2018\end{array}$ & $\begin{array}{l}\text { Variación } \\
2018 / 2017\end{array}$ & $\begin{array}{l}\text { Ciencias } \\
\text { Sociales }\end{array}$ & Educación & $\begin{array}{l}\text { Ingeniería, } \\
\text { Ciencia y }\end{array}$ & Salud \\
\hline Prestigio y Lealtad & 5,31 & 5,23 & $-1,6 \%$ & 5,34 & 5,46 & 5,33 & 5,11 \\
\hline $\begin{array}{l}\text { 1. Considerando tu experiencia como } \\
\text { alumno de la institución y considerando } \\
\text { una escala de } 1 \text { a } 7: \text { ¿Qué tan } \\
\text { satisfecho estás con la UBO? }\end{array}$ & 5,42 & 5,32 & $-1,9 \%$ & 5,38 & 5,57 & 5,53 & 5,20 \\
\hline $\begin{array}{l}\text { 2. Siempre pensando en estos } 2 \text { últimos } \\
\text { años: ¿Dirías que la Infraestructura de } \\
\text { la UBO ha mejorado? }\end{array}$ & 5,18 & $\mathbf{5 , 2 7}$ & $1,7 \%$ & 5,41 & 5,29 & 5,07 & 5,26 \\
\hline $\begin{array}{l}\text { 3. A tu juicio y en términos generales } \\
\text { ¿Dirías que estos } 2 \text { últimos años la } \\
\text { UBO ha mejorado? }\end{array}$ & 5,29 & 5,28 & $-0,2 \%$ & 5,39 & 5,40 & 5,26 & 5,22 \\
\hline $\begin{array}{l}\text { 4. Si algún amigo te pidiera que le } \\
\text { recomendaras una universidad: ¿Cuán } \\
\text { dispuesto estarías a recomendar a tus } \\
\text { amigos que estudien en la UBO? }\end{array}$ & 5,40 & 5,28 & $-2,1 \%$ & 5,38 & 5,59 & 5,44 & 5,14 \\
\hline $\begin{array}{l}\text { 5. Si en este momento tuvieras la } \\
\text { posibilidad de volver a elegir una } \\
\text { Universidad para estudiar: ¿Cuán } \\
\text { dispuesto estarías en volver a elegir a } \\
\text { UBO? }\end{array}$ & 5,25 & 5,10 & $-2,9 \%$ & 5,23 & 5,50 & 5,28 & 4,91 \\
\hline $\begin{array}{l}\text { 6. ¿Cómo calificarías la siguiente } \\
\text { afirmación? La UBO es una institución } \\
\text { seria y confiable }\end{array}$ & 5,49 & 5,35 & $-2,6 \%$ & 5,47 & 5,56 & 5,58 & 5,22 \\
\hline $\begin{array}{l}\text { 7. ¿Cómo calificarías la siguiente } \\
\text { afirmación? La UBO es Prestigiosa }\end{array}$ & 5,13 & 4,97 & $-3,2 \%$ & 5,10 & 5,29 & 5,12 & 4,81 \\
\hline
\end{tabular}

Fuente: Elaboración propia. 
La Tabla 2 es el resultado de la variable Servicios, variable principal del estudio, donde nuevamente, los estudiantes de la Facultad de Salud presentan la percepción más baja, y algunos servicios como alimentación y pago en cajas recaudadoras presentan percepción promedio inferior a 5.0; en este caso la percepción no ha variado de un año a otro de manera general, sí en algunos indicadores medidos.

Tabla 2. Variable Servicios UBO 2017 y 2018.

\begin{tabular}{|c|c|c|c|c|c|c|c|}
\hline Preguntas & $\begin{array}{l}\text { UBO } \\
2017\end{array}$ & $\begin{array}{l}\text { UBO } \\
2018\end{array}$ & $\begin{array}{l}\text { Variación } \\
\text { 2018/2017 }\end{array}$ & $\begin{array}{l}\text { Ciencias } \\
\text { Sociales }\end{array}$ & Educación & $\begin{array}{c}\text { Ingeniería, } \\
\text { Ciencia y } \\
\text { Tecnología }\end{array}$ & Salud \\
\hline Servicios & 5,09 & 5,09 & $0,0 \%$ & 5,12 & 5,28 & 5,18 & 5,01 \\
\hline $\begin{array}{l}\text { 39. ¿Qué tan satisfecho estás con los } \\
\text { servicios de alimentación? }\end{array}$ & 4,69 & 4,81 & $2,6 \%$ & 4,79 & 5,19 & 5,08 & 4,66 \\
\hline $\begin{array}{l}\text { 40. ¿Qué tan satisfecho estás con los } \\
\text { servicios higiénicos? }\end{array}$ & 5,32 & 5,36 & $0,9 \%$ & 5,42 & 5,61 & 5,50 & 5,25 \\
\hline $\begin{array}{l}\text { 41. ¿Qué tan satisfecho estás con los } \\
\text { servicios de recreación? }\end{array}$ & 5,13 & 5,15 & $0,4 \%$ & 5,14 & 5,39 & 5,26 & 5,08 \\
\hline $\begin{array}{l}\text { 42. ¿Qué tan satisfecho estás con el servicio } \\
\text { de fotocopiadora? }\end{array}$ & 5,24 & 5,26 & $0,4 \%$ & 5,36 & 5,30 & 5,24 & 5,22 \\
\hline $\begin{array}{l}\text { 43. ¿Qué tan satisfecho estás con el servicio } \\
\text { de cajas recaudadoras? }\end{array}$ & 5,08 & 4,87 & $-4,3 \%$ & 4,92 & 4,92 & 4,81 & 4,85 \\
\hline $\begin{array}{l}\text { 44. Considerando ahora todos estos } \\
\text { aspectos que hemos revisado, ¿cuál es tu } \\
\text { nivel de satisfacción con la UBO en general } \\
\text { como institución educativa? }\end{array}$ & 5,36 & 5,25 & $-1,9 \%$ & 5,30 & 5,47 & 5,34 & 5,17 \\
\hline
\end{tabular}

Fuente: Elaboración propia.

Tabla 3. Otras Variables de servicios UBO 2017 y 2018.

\begin{tabular}{|l|c|c|c|c|c|c|c|}
\hline \multicolumn{1}{|c|}{ Preguntas } & $\begin{array}{l}\text { UBO } \\
\mathbf{2 0 1 7}\end{array}$ & $\begin{array}{c}\text { UBO } \\
\mathbf{2 0 1 8}\end{array}$ & $\begin{array}{c}\text { Variación } \\
\mathbf{2 0 1 8} / \mathbf{2 0 1 7}\end{array}$ & $\begin{array}{l}\text { Ciencias } \\
\text { Sociales }\end{array}$ & $\begin{array}{c}\text { Ingeniería, } \\
\text { Ciencia y } \\
\text { Tecnología }\end{array}$ & Salud \\
\hline Instalaciones & $\mathbf{5 , 5 2}$ & $\mathbf{5 , 4 9}$ & $\mathbf{- 0 , 6 \%}$ & $\mathbf{5 , 5 5}$ & $\mathbf{5 , 6 3}$ & $\mathbf{5 , 4 1}$ & $\mathbf{5 , 4 5}$ \\
\hline $\begin{array}{l}\text { 31. Las instalaciones de la universidad son } \\
\text { adecuadas. }\end{array}$ & $\mathbf{5 , 4 4}$ & $\mathbf{5 , 3 8}$ & $\mathbf{- 1 , 0 \%}$ & $\mathbf{5 , 4 3}$ & 5,53 & 5,41 & 5,33 \\
\hline $\begin{array}{l}\text { 32. La implementación de laboratorios (sala de } \\
\text { simulación, computación y otros) es apropiada } \\
\text { para la docencia. }\end{array}$ & $\mathbf{5 , 5 4}$ & $\mathbf{5 , 4 7}$ & $\mathbf{- 1 , 3 \%}$ & $\mathbf{5 , 5 4}$ & 5,64 & 5,28 & 5,44 \\
\hline $\begin{array}{l}\text { 33. Existe disponibilidad de equipamiento en las } \\
\text { instalaciones (PC, Data, Pizarra, etc.). }\end{array}$ & $\mathbf{5 , 7 1}$ & $\mathbf{5 , 7 4}$ & $\mathbf{0 , 6 \%}$ & $\mathbf{5 , 8 2}$ & 5,84 & 5,62 & 5,71 \\
\hline $\begin{array}{l}\text { 34. iCómo calificarías la infraestructura de las } \\
\text { salas de clases? }\end{array}$ & $\mathbf{5 , 4 1}$ & $\mathbf{5 , 3 7}$ & $\mathbf{- 0 , 7 \%}$ & $\mathbf{5 , 4 0}$ & 5,50 & 5,34 & 5,33 \\
\hline Comunicaciones & $\mathbf{5 , 3 5}$ & $\mathbf{5 , 1 2}$ & $\mathbf{- 4 , 3 \%}$ & $\mathbf{5 , 2 1}$ & $\mathbf{5 , 3 5}$ & $\mathbf{5 , 2 6}$ & $\mathbf{5 , 0 1}$ \\
\hline $\begin{array}{l}\text { 35. iCómo calificarías la página web y redes } \\
\text { sociales de la universidad? }\end{array}$ & $\mathbf{5 , 5 0}$ & $\mathbf{5 , 4 2}$ & $\mathbf{- 1 , 4 \%}$ & $\mathbf{5 , 4 5}$ & 5,59 & 5,43 & 5,36 \\
\hline $\begin{array}{l}\text { 36. iCómo calificarías el call center o atención } \\
\text { telefónica en general? }\end{array}$ & $\mathbf{5 , 2 1}$ & $\mathbf{4 , 8 3}$ & $\mathbf{- 7 , 3 \%}$ & $\mathbf{4 , 9 7}$ & 5,11 & 5,09 & 4,66 \\
\hline Matrícula & $\mathbf{5 , 4 4}$ & $\mathbf{5 , 3 0}$ & $\mathbf{- 2 , 6 \%}$ & $\mathbf{5 , 3 9}$ & $\mathbf{5 , 4 9}$ & $\mathbf{5 , 4 0}$ & $\mathbf{5 , 2 0}$ \\
\hline $\begin{array}{l}\text { 37. El proceso de matrícula es fluido, expedito y } \\
\text { sin tramitación excesiva. }\end{array}$ & $\mathbf{5 , 3 6}$ & $\mathbf{5 , 1 5}$ & $\mathbf{- 4 , 0 \%}$ & $\mathbf{5 , 2 5}$ & 5,35 & 5,28 & 5,04 \\
\hline $\begin{array}{l}\text { 38. Los requisitos de admisión de alumnos a la } \\
\text { Universidad son conocidos por los postulantes. }\end{array}$ & $\mathbf{5 , 5 1}$ & $\mathbf{5 , 4 5}$ & $\mathbf{- 1 , 2 \%}$ & $\mathbf{5 , 5 3}$ & 5,63 & 5,52 & 5,36 \\
\hline
\end{tabular}

Fuente: Elaboración propia. 
La Tabla 3 detalla los resultados de otras variables de servicio del estudio, todas con una disminución de la percepción de satisfacción, por lo que confirma la importancia del modelo creado, que busca las relaciones de todas estas variables, buscando mejorar la percepción estudiantil, desde medidas que apunten a mejorar desde las indicaciones que pueda entregar el modelo creado.

Finalmente, la Tabla 4 muestra las percepciones de las variables de docencia, las mejor evaluadas por los estudiantes, destacando los servicios de biblioteca, formación integral y los docentes.

Tabla 4. Variables de Docencia UBO 2017 y 2018.

\begin{tabular}{|c|c|c|c|c|c|c|c|}
\hline Preguntas & \begin{tabular}{|l|} 
UBO \\
2017 \\
\end{tabular} & $\begin{array}{l}\text { UBO } \\
2018 \\
\end{array}$ & $\begin{array}{r}\text { Variación } \\
2018 / 2017 \\
\end{array}$ & \begin{tabular}{|l} 
Ciencias \\
Sociales \\
\end{tabular} & Educación & \begin{tabular}{|c|} 
Ingeniería, \\
Ciencia y \\
Tecnología \\
\end{tabular} & Salud \\
\hline Formación Integral & 5,50 & 5,52 & $0,2 \%$ & 5,52 & 5,65 & 5,63 & 5,46 \\
\hline $\begin{array}{l}\text { 14. La información y accesibilidad respecto de } \\
\text { programas de apoyo de formación integral para } \\
\text { los estudiantes (nivelación, reforzamiento, apoyo } \\
\text { psicoeducativo y talleres de apoyo) es accesible } \\
\text { para los estudiantes. }\end{array}$ & 5,53 & 5,55 & $0,3 \%$ & 5,56 & 5,67 & 5,65 & 5,50 \\
\hline $\begin{array}{l}\text { 15. Los programas de apoyo de formación } \\
\text { integral colaboran a la mejora de los aprendizajes. }\end{array}$ & 5,48 & 5,48 & $0,0 \%$ & 5,49 & 5,63 & 5,61 & 5,41 \\
\hline Biblioteca & 5,66 & 5,64 & $-0,3 \%$ & 5,64 & 5,84 & 5,69 & 5,58 \\
\hline 16. ¿Cómo calificarías la atención en biblioteca? & 5,62 & 5,61 & $-0,3 \%$ & 5,65 & 5,85 & 5,77 & 5,50 \\
\hline 17. Los horarios de atención en la biblioteca. & 5,76 & 5,74 & $-0,3 \%$ & 5,72 & 5,90 & 5,72 & 5,71 \\
\hline $\begin{array}{l}\text { 18. La bibliografía de las asignaturas que cursó es } \\
\text { actualizada. }\end{array}$ & 5,59 & 5,58 & $-0,2 \%$ & 5,56 & 5,77 & 5,57 & 5,53 \\
\hline Docencia & 5,46 & 5,34 & $-2,2 \%$ & 5,39 & 5,60 & 5,43 & 5,23 \\
\hline $\begin{array}{l}\text { 19. He podido verificar que mi proceso de } \\
\text { aprendizaje es coherente con lo que estipula el } \\
\text { Modelo de Formación. }\end{array}$ & 5,52 & 5,48 & $-0,8 \%$ & 5,53 & 5,69 & 5,56 & 5,39 \\
\hline $\begin{array}{l}20 . \quad \text { La evaluación docente es útil para } \\
\text { retroalimentar la calidad de la formación de los } \\
\text { alumnos. }\end{array}$ & 5,47 & 5,45 & $-0,5 \%$ & 5,45 & 5,64 & 5,52 & 5,38 \\
\hline $\begin{array}{l}\text { 21. El sistema de información intranet es útil y } \\
\text { óptimo para consultas académicas y trámites de } \\
\text { los alumnos (inscripción de asignaturas, consulta } \\
\text { de notas, otras). }\end{array}$ & 5,55 & 5,37 & $-3,4 \%$ & 5,46 & 5,65 & 5,44 & 5,24 \\
\hline $\begin{array}{l}\text { 22. La respuesta a solicitudes administrativas de } \\
\text { los alumnos (certificados, inscripción de } \\
\text { asignaturas, otras) es oportuna. }\end{array}$ & 5,28 & 5,06 & $-4,3 \%$ & 5,13 & 5,41 & 5,20 & 4,91 \\
\hline Profesores & 5,60 & 5,58 & $-0,2 \%$ & 5,62 & 5,85 & 5,68 & 5,48 \\
\hline $\begin{array}{l}\text { 23. Las metodologías de enseñanza facilita mi } \\
\text { aprendizaje. }\end{array}$ & 5,48 & 5,44 & $-0,8 \%$ & 5,52 & 5,74 & 5,49 & 5,32 \\
\hline $\begin{array}{l}\text { 24. Existe claridad y difusión de los criterios de } \\
\text { evaluación que utiliza el profesor. }\end{array}$ & 5,48 & 5,47 & $-0,2 \%$ & 5,50 & 5,71 & 5,55 & 5,38 \\
\hline $\begin{array}{l}\text { 25. En la Universidad es posible establecer } \\
\text { relaciones cordiales con profesores. }\end{array}$ & 5,79 & 5,79 & $-0,1 \%$ & 5,83 & 6,00 & 5,90 & 5,69 \\
\hline $\begin{array}{l}\text { 26. El profesor se preocupa por dar horas de } \\
\text { atención para plantear consultas y dudas sobre las } \\
\text { materias del curso. }\end{array}$ & 5,57 & 5,56 & $-0,1 \%$ & 5,60 & 5,87 & 5,69 & 5,44 \\
\hline $\begin{array}{l}\text { 27. El profesor entrega material de apoyo } \\
\text { académico (guías, ejercicio, lecturas etc.) que } \\
\text { facilitan el aprendizaje. }\end{array}$ & 5,67 & 5,66 & $-0,1 \%$ & 5,66 & 5,93 & 5,76 & 5,57 \\
\hline Directivos de Escuelas & 5,26 & 5,14 & $-2,3 \%$ & 5,20 & 5,69 & 5,43 & 4,92 \\
\hline $\begin{array}{l}\text { 28. Existe disponibilidad de las autoridades de la } \\
\text { carrera para las consultas de los estudiantes. }\end{array}$ & 5,38 & 5,29 & $-1,7 \%$ & 5,29 & 5,81 & 5,53 & 5,10 \\
\hline
\end{tabular}


29. Existe cercanía de las autoridades con los alumnos.

30. Las autoridades manifiestan capacidad para resolver los problemas de los alumnos.

\begin{tabular}{|c|c|c|c|c|c|c|}
$\mathbf{5 , 1 8}$ & $\mathbf{5 , 0 7}$ & $\mathbf{- 2 , 2 \%}$ & 5,15 & 5,64 & 5,38 & 4,83 \\
\hline $\mathbf{5 , 2 2}$ & $\mathbf{5 , 0 6}$ & $\mathbf{- 3 , 1 \%}$ & 5,15 & 5,63 & 5,38 & 4,82 \\
\hline
\end{tabular}

Fuente: Elaboración propia.

\subsection{Análisis estadístico de la encuesta de satisfacción.}

El siguiente análisis de la encuesta de satisfacción de la Universidad Bernardo O'Higgins fue realizado para los años 2017 y 2018 a 4.386 y 4.646 alumnos (estudio censal), respectivamente. En dicha encuesta se realizaron 44 preguntas evaluadas de forma cuantitativa, con calificaciones del 1 al 7 y una pregunta en la que se pide explicar el porqué de las calificaciones a cada alumno encuestado.

El análisis estadístico se realizó a las 44 preguntas calificadas de forma cuantitativa mediante análisis factorial, que estudia las variables observadas en los individuos, generando correlaciones y calculando parámetros que permiten la representación de variables latentes, que son combinaciones de ciertas variables observadas y que podrían generar otro carácter observable o de interés de estudio para los datos analizados.

Específicamente, en el análisis se trabajó con la matriz de correlaciones, estadísticos que indican varianza absorbida a nivel general por los factores generados, comunalidades (varianza absorbida por cada variable al buscar variables latentes), matriz componente o estructura factorial que representa la saturación de cada variable en el o los factores encontrados y las pruebas KMO y de esfericidad de Bartlett.

Existen varios contrastes que pueden realizarse para evaluar si el modelo factorial (o extracción de los factores) tiene significancia.

El test KMO (Kaiser, Meyer y Olkin) relaciona los coeficientes de correlación observados entre las variables. Cuanto más cerca de 1 sea el valor obtenido del test $\mathrm{KMO}$, la relación entre las variables es alta. Un KMO mayor o igual a 0,9 considera un test muy bueno; mayor a 0,8 un test notable, mediano si es mayor a 0,7 , bajo si es mayor que 0,6 y muy bajo si es menor que 0,5 .

La prueba de esfericidad de Bartlett evalúa la aplicabilidad del análisis factorial de las variables estudiadas. Para esta prueba se aceptan valores menores a 0,05 para que el análisis factorial tenga sentido.

En la Tabla 5 se observa que los estadísticos antes mencionados cumplen con valores muy favorables para el análisis factorial. La medida KMO en las encuestas de satisfacción del 2017 y 2018 superan el 0,98 y la prueba de esfericidad de Bartlett está por debajo del 0,05 antes mencionado.

Tabla 5. Medidas KMO y Bartlett.

\begin{tabular}{|l|l|r|r|}
\hline \multicolumn{2}{|c|}{ Prueba de KMO y Bartlett } & UBO 2017 & \multicolumn{1}{c|}{ UBO 2018} \\
\hline $\begin{array}{l}\text { Medida Kaiser-Meyer-Olkin de adecuación de } \\
\text { muestreo }\end{array}$ & & 0,988 & 0,987 \\
\hline \multirow{2}{*}{ Prueba de esfericidad de Bartlett } & $\begin{array}{l}\text { Aprox. Chi- } \\
\text { cuadrado }\end{array}$ & $184.965,746$ & $190.172,555$ \\
\hline & Gl & 946 & 946 \\
\cline { 2 - 4 } & Sig. & $\mathbf{0 , 0 0 0}$ & $\mathbf{0 , 0 0 0}$ \\
\hline
\end{tabular}

Fuente: Elaboración propia. 
Por lo tanto, tiene sentido hacer el análisis factorial y este modelo es adecuado para explicar los datos. En la Tabla 6 se muestran los valores singulares y la absorción de varianza para los primeros 4 ejes factoriales.

Tabla 6. Autovalores y Absorción de Varianza.

\begin{tabular}{|c|c|c|r|r|r|r|}
\hline $\begin{array}{c}\text { Componente } \\
\text { factorial }\end{array}$ & $\begin{array}{c}\text { Total } \\
2017\end{array}$ & $\begin{array}{c}\text { Total } \\
\mathbf{2 0 1 8}\end{array}$ & $\begin{array}{c}\text { \% de } \\
\text { varianza } \\
\mathbf{2 0 1 7}\end{array}$ & $\begin{array}{c}\text { \% de } \\
\text { varianza } \\
\mathbf{2 0 1 8}\end{array}$ & $\begin{array}{c}\text { \% acumulado } \\
\mathbf{2 0 1 7}\end{array}$ & $\begin{array}{c}\text { \% acumulado } \\
\mathbf{2 0 1 8}\end{array}$ \\
\hline 1 & $\mathbf{2 6 , 1 4 6}$ & $\mathbf{2 5 , 3 8 9}$ & $\mathbf{5 9 , 4 2 2}$ & $\mathbf{5 7 , 7 0 2}$ & $\mathbf{5 9 , 4 2 2}$ & $\mathbf{5 7 , 7 0 2}$ \\
\hline 2 & 1,532 & 1,639 & $\mathbf{3 , 4 8 2}$ & $\mathbf{3 , 7 2 5}$ & $\mathbf{6 2 , 9 0 3}$ & $\mathbf{6 1 , 4 2 7}$ \\
\hline 3 & 1,348 & 1,511 & $\mathbf{3 , 0 6 4}$ & $\mathbf{3 , 4 3 5}$ & $\mathbf{6 5 , 9 6 7}$ & $\mathbf{6 4 , 8 6 2}$ \\
\hline 4 & 1,193 & 1,232 & $\mathbf{2 , 7 1 1}$ & $\mathbf{2 , 7 9 9}$ & $\mathbf{6 8 , 6 7 9}$ & $\mathbf{6 7 , 6 6 2}$ \\
\hline
\end{tabular}

Fuente: Elaboración propia.

De acuerdo a la información, se observa que en el primer eje factorial se absorbe casi un $60 \%$ de la varianza total de los datos para los años 2017 y 2018, por lo tanto, esta componente es la variable latente en el análisis. Es decir, una variable observable o que podría explicar alguna característica de interés en nuestro conjunto de datos. Además, se observa que desde la segunda componente en adelante la varianza absorbida por las mismas es menor al 4\%; debido a esto la representación gráfica, o proyección mediante los ejes factoriales, no será de interés en este estudio ya que no representará de manera fidedigna la distribución de datos ni relación entre variables; además, dichas componentes no serán buenos candidatos a variables latentes.

En la Tabla 7 se presentan las comunalidades de cada variable en el análisis factorial realizado. Se observa que casi todas las variables participantes del análisis factorial están representadas con más de un $60 \%$ de confiabilidad.

Tabla 7. Comunalidades.

\begin{tabular}{|l|r|r|l|l|r|r|}
\hline & Extracción 2017 & Extracción 2018 & & Extracción 2017 & Extracción 2018 \\
\hline P1 & 694 & 702 & P23 & 692 & 681 \\
\hline P2 & 611 & 600 & $\mathbf{P 2 4}$ & 683 & 690 \\
\hline P3 & 705 & 708 & $\mathbf{P 2 5}$ & 737 & 747 \\
\hline P4 & 775 & 793 & $\mathbf{P 2 6}$ & 696 & 688 \\
\hline P5 & 759 & 764 & $\mathbf{P 2 7}$ & 712 & 696 \\
\hline P6 & 756 & 764 & $\mathbf{P 2 8}$ & 825 & 816 \\
\hline P7 & 730 & 723 & $\mathbf{P 2 9}$ & 866 & 831 \\
\hline P8 & 593 & 545 & $\mathbf{P 3 0}$ & 868 & 836 \\
\hline P9 & 734 & 707 & $\mathbf{P 3 1}$ & 671 & 658 \\
\hline P10 & 688 & 673 & $\mathbf{P 3 2}$ & 664 & 652 \\
\hline P11 & 744 & 746 & $\mathbf{P 3 3}$ & 688 & 697 \\
\hline P12 & 513 & 517 & $\mathbf{P 3 4}$ & 683 & 682 \\
\hline P13 & 577 & 629 & $\mathbf{P 3 5}$ & 704 & 657 \\
\hline P14 & 667 & 659 & $\mathbf{P 3 6}$ & 646 & 657 \\
\hline P15 & 690 & 693 & $\mathbf{P 3 7}$ & 644 & 648 \\
\hline P16 & 610 & 596 & $\mathbf{P 3 8}$ & 678 & 667 \\
\hline P17 & 691 & 656 & $\mathbf{P 3 9}$ & 685 & 648 \\
\hline P18 & 682 & 677 & $\mathbf{P 4 0}$ & 586 & 549 \\
\hline P19 & 741 & 750 & $\mathbf{P 4 1}$ & 690 & 659 \\
\hline P20 & 584 & 580 & $\mathbf{P 4 2}$ & 622 & 595 \\
\hline P21 & 641 & 583 & $\mathbf{P 4 3}$ & 663 & 633 \\
\hline P22 & 576 & 561 & $\mathbf{P 4 4}$ & 756 & 759 \\
\hline & & Flabor & & \\
\hline
\end{tabular}

Fuente: Elaboración propia. 
Por último, en la Tabla 8 se exhibe la matriz de estructura factorial que refleja el peso de cada variable en cada componente. Por lo mencionado anteriormente, este estudio se centrará solo en la primera componente ya que es la que absorbe más varianza.

Tabla 8. Estructura factorial para primera componente.

\begin{tabular}{|l|r|r|l|l|r|r|}
\hline & Factor 2017 & Factor 2018 & & Factor 2017 & Factor 2018 \\
\hline P1 & 780 & 777 & & $\mathbf{P 2 3}$ & 824 & 813 \\
\hline P2 & 674 & 683 & & $\mathbf{P 2 4}$ & 815 & 807 \\
\hline P3 & 762 & 758 & & $\mathbf{P 2 5}$ & 790 & 783 \\
\hline P4 & 790 & 790 & $\mathbf{P 2 6}$ & 790 & 771 \\
\hline P5 & 768 & 765 & & $\mathbf{P 2 7}$ & 786 & 770 \\
\hline P6 & 813 & 811 & $\mathbf{P 2 8}$ & 748 & 746 \\
\hline P7 & 777 & 754 & & $\mathbf{P 2 9}$ & 730 & 722 \\
\hline P8 & 727 & 695 & & $\mathbf{P 3 0}$ & 758 & 741 \\
\hline P9 & 811 & 797 & & $\mathbf{P 3 1}$ & 796 & 766 \\
\hline P10 & 796 & 795 & & $\mathbf{P 3 2}$ & 793 & 765 \\
\hline P11 & 830 & 831 & & $\mathbf{P 3 3}$ & 779 & 764 \\
\hline P12 & 714 & 718 & & $\mathbf{P 3 4}$ & 781 & 771 \\
\hline P13 & 745 & 741 & $\mathbf{P 3 5}$ & 810 & 782 \\
\hline P14 & 790 & 780 & & $\mathbf{P 3 6}$ & 747 & 673 \\
\hline P15 & 814 & 811 & $\mathbf{P 3 7}$ & 762 & 729 \\
\hline P16 & 733 & 724 & $\mathbf{P 3 8}$ & 806 & 799 \\
\hline P17 & 737 & 719 & $\mathbf{P 3 9}$ & 634 & 671 \\
\hline P18 & 794 & 780 & $\mathbf{P 4 0}$ & 708 & 692 \\
\hline P19 & 846 & 848 & $\mathbf{P 4 1}$ & 758 & 758 \\
\hline P20 & 759 & 749 & $\mathbf{P 4 2}$ & 699 & 699 \\
\hline P21 & 783 & 759 & $\mathbf{P 4 3}$ & 691 & 662 \\
\hline P22 & 749 & 728 & $\mathbf{P 4 4}$ & 860 & 863 \\
\hline
\end{tabular}

Fuente: Elaboración propia.

En esta estructura factorial se presenta la saturación relativa de cada variable en el primer factor del análisis factorial. Se observa que todas las variables saturan al menos un $60 \%$ la primera componente, es decir, todas las variables (preguntas de la encuesta de satisfacción) son partícipes de la candidata a variable latente, que podría denominarse calidad (en concordancia con el estudio). O como explica el estudio Prestigio y Lealtad. Los factores de la Tabla 8, son los coeficientes $\beta$ del modelo construido en el diagrama 1 con lo que el modelo de relaciones de variables queda significativamente establecido.

\section{Conclusiones.}

En las últimas décadas, los cambios y tensiones que han debido enfrentar las instituciones de educación superior las han llevado a replantearse su rol como agentes de formación y promoción del desarrollo social. Es así como, con fuerte dinamismoy, han debido asumir desde la expansión de la cobertura de sus matrículas, hasta la diversificación del sistema educativo, lo que las ha interpelado a ser más competitivas.

En este contexto, la presente investigación, en esta primera etapa, ha tenido como objetivo modelar un sistema derivado del comportamiento de respuestas del consumidor educativo de la Universidad Bernardo O'Higgins. La razón de la investigación responde a una falta de estudios en el 
sector de servicios de la educación terciaria. El método sugerido para modelar contrastar variables es un aporte metodológico dada la escasez de investigaciones, que intenta responder las hipótesis generadas en el estudio. En la segunda parte de esta investigación se entregarán los resultados derivados de la aplicación de este modelo en el año 2019 y 2020, que desde el punto de vista estadístico tienen varias ventajas, tales como:

- Mayor flexibilidad estadística (permite interpretación incluso en presencia de multicolinealidad).

- Uso del Análisis Factorial Confirmatorio para reducir el error de medida (múltiples indicadores por variable latente).

- Posibilidad de contrastar modelos conjuntamente.

- Interfaz de representación gráfica de SEM.

Este modelo permite:

- Contrastar modelos con múltiples variables dependientes.

- Incorporar variables mediadoras.

- Modelizar y medir los errores.

- Comparar el modelo para submuestras diferentes (Multimuestra).

En las predicciones del modelo que serán realizados en los dos siguientes años, es prudente considerar la matriz de correlaciones del modelo dado, ya que variables por debajo de un 0,75 de confiabilidad pueden no ser consideradas en el Diagrama 1.

Se espera que los resultados arrojados en este estudio se transformen en importantes insumos a la hora de replantear los procesos de gestión institucional de la Universidad en las áreas prioritarias referentes a docencia, servicios, administración, dirección, formación integral, entre otras, que repercuten de manera directa en el grado de satisfacción de sus estudiantes.

Según lo descrito en esta investigación, lograr mejoras en la calidad de los servicios entregados por la Universidad será posible solo si se entiende y valora los procesos de gestión de calidad como principios cardinales donde todos los que forman parte de la institución son corresponsables y además, están dispuestos al diálogo necesario para establecer una caracterización de que la institución entenderá por calidad, considerando las perspectivas de todos sus actores, especialmente, la de sus estudiantes.

Bajo estas consideraciones y en función de la experiencia del estudiante es recomendable centrar con especial interés las mejoras de gestión en los elementos involucrados en tres momentos de la vida de los estudiantes, los que de algún modo u otro son explicitados a través de la encuesta de satisfacción estudiantil. El primero, en el ingreso procurando su integración, lo que implica el acceso oportuno a la información, acompañamiento y orientación en los procesos administrativos, acceso a instancias de formación y apoyo integral, para asegurar la permanencia de los estudiantes. El segundo, en su proceso de formación, evaluando la efectividad de las estrategias educativas, los métodos pedagógicos y el desarrollo de las competencias generales de su formación profesional en coherencia con el perfil de egresado de cada carrera. Finalmente, en su egreso considerando la titulación oportuna y los factores de empleabilidad e incorporación al mercado laboral.

Por último, se ha de destacar que el manejo y análisis de la información que ha emergido de estudio plantea que la factibilidad de implementar una gestión más resolutiva y eficiente requiere fortalecer los mecanismos sistemáticos de control de calidad de cada uno de los procesos institucionales. Del mismo modo, es necesario sensibilizar que el análisis de cada uno de los elementos estudiados en esta investigación implica integrar cada una de sus variables para su uso en la toma de decisiones. 


\section{Referencias}

Arambewela, R., \& Hall, J. (2006). A comparative analysis of international education satisfaction using SERVQUAL. Journal of Services Research, 6, 141-163.

Blanco-Guillermety, R. J., \& Blanco-Peck, R. (2018). La medición de la calidad de servicios en la educación universitaria. Revista de Educación de Puerto Rico (REduca), 22, 121-136.

Beltyukova, SA \& Fox, CM. (2002). La satisfacción del estudiante como medida del desarrollo del estudiante: hacia una métrica universal. Journal of College Student Development, 43 (2), 161-172.

Bigné, J., Moliner, M., Vallet, T., \& Sánchez, J. (1997). Un Estudio Comparativo de los Instrumentos de Medición de la Calidad de los Servicios Públicos. Revista Española de Investigación de Marketing-ESIC, 1, 33-53.

Cabrera, V. (2005). El concepto calidad en la educación universitaria: clave para el logro de la competitividad institucional. Revista iberoamericana de Educación, 36(12), 1-7.

Camisón, C., Gil, M., \& Roca, V. (1999). Hacia Modelos de Calidad de Servicio Orientados al Cliente en las Universidades Públicas: el Caso de la Universitat Jaume I. Investigaciones Europeas de Dirección y Economía de la Empresa, 5(2), 69-92.

Capelleras, J., \& Veciana, J. (2004). Calidad de Servicio en la Enseñanza Universitaria: Desarrollo y Validación de una Escala de Medida. Revista Europea de Dirección y Economía de la Empresa, 13(4), 55-72.

Chin, W. (1998). Commentary: Issues and Opinion on Structural Equation Modeling. MIS Quarterly, 22(1), VII-XVI.

Chung, E. \& McLarney, C. (2000). The classroom as a service encounter: Suggestions for value creation. Journal of Management Education, 24(4), 484-500.

Cronin Jr, J., Brady, M., \& Hult, G. (2000). Assessing the effects of quality, value, and customer satisfaction on consumer behavioral intentions in service environments. Journal of retailing, 76(2), 193-218.

De la Fuente, H., Marzo, M., Riquelme, R. \& Jesús, M. (2010). Análisis de la satisfacción universitaria en la Facultad de Ingeniería de la Universidad de Talca. Ingeniare. Revista chilena de ingeniería, 18(3), 350-363.

Gaitán, J., \& López, M. (1999). La calidad, nueva función en la Universidad Venezolana. La Revista de Investigación y Postgrado DOCTUM, 3(1), 285-300.

Grönroos, C. (1994). Marketing y gestión de servicios: la gestión de los momentos de la verdad y la competencia en los servicios. Madrid: Ediciones Díaz de Santos.

Hermida, M., Buendía, L., \& López, R. (2008). La calidad en el contexto universitario. Contribuciones a las Ciencias Sociales, (2008-01). Recuperado de http://www. eumed. net/rev/cccss. 
Joseph, M. \& Joseph, B. (1997). Service Quality in Education: A Student Perspective. Quality in Assurance in Education, 5(1), 15-2.

Kwan, P. \& Ng, P. (1999). Quality Indicators in Higher Education-Comparing Hong Kong and China's Students. Managerial Auditing Journal, 14(1), 20-27.

Lagarda, A. (2001). La educación superior y el mercado de trabajo profesional. REDIE: Revista Electrónica de Investigación Educativa, 3(1), 55-66.

LeBlanc, G. \& Nguyen, N. (1997). Searching for excellence in Business Education: An Exploratory Study of Customer Impressions of Service Quality. International Journal of Educational Management, 11(2), 72-79.

Lepeley, M. (2001). Gestión y calidad en educación: un modelo de evaluación. México: McGraw-Hill Interamericana.

Mintzberg, H., Nieto, J., \& Bonner, D. (1991). La naturaleza del trabajo directivo. España: Ariel.

Morresi, S., Donnini, N., \& Cerioni, L. L. (2008). Indicadores de calidad en la evaluación de instituciones de educación superior. Algunas consideraciones críticas. VIII Coloquio Internacional sobre Gestión Universitaria de América del Sur. Asunción, Paraguay. Recuperado de https://repositorio.ufsc.br/handle/123456789/61508

Oliver, R.L. (1980). A cognitive model of the antecedents and consequences of satisfaction decisions. Journal of Marketing Research, 17 (4), 460-469.

Owlia, M., \& Aspinwall, E. (1996). Quality in higher education: a survey. Total Quality Management, 7(2), 161-171.

Parasuraman, A., Zeithaml, V., \& Berry, L. (1985). A Conceptual Model of Service Quality and Its Implications for Future Research. Journal of Marketing, 49 (4), 41-50.

Salmi, J., (2009). El desafio de crear universidades de rango mundial. Bogotá: Banco MundialMayol Ediciones.

Salvador, C. (2010). Quality of university services: Dimensional structure of SERVQUAL vs. ESQS. Service Science, 2(3), 167-176.

Shekarchizadeh, A., Rasli, A. \& Hon-Tat, H. (2011). SERVQUAL in Malaysian universities: perspectives of international students. Business Process Management Journal, 17(1), 6781.

Sverdlick, I. (2012). Calidad de educación para todos e inclusión educativa ¿Nuevas banderas para el derecho a la educación? Revista Internacional Magisterio, 56, 50-55. 
Tünnermann, C. (2006). Pertinencia y calidad de la educación superior. Lección inaugural. Guatemala. Recuperado de https://www.researchgate.net/profile/Luis-GonzalezFiegehen/publication/309493290_La_educacion_superior_en_Chile_en_revista_Pensamie nto_Universitario_2011_-_10/links $/ 5813978 \mathrm{f08a}$ aeffbed6bc23ea/La-educacion-superioren-Chile-en-revista-Pensamiento-Universitario-2011-10.pdf\#page $=95$

Universidad Bernardo O'Higgins (UBO) (2018). Sistema de aseguramiento de la calidad. Recuperado de http://www.ubo.cl/dgdes/aseguramiento-la-calidad/

Vázquez, M., Elizondo, A., \& Ramírez, J. (2017). Calidad de los servicios universitarios: Una percepción desde sus actores en una escuela de negocios. Innovaciones de negocios, 13(25), 71-95.

Westbrook, R. (1987). Product/consumption-based affective responses and postpurchase processes. Journal of Marketing Research, 24(3), 258-270.

Zineldin, M., Akdag, H. \& Vasicheva, V. (2011). Assessing quality in higher education: New criteria for evaluating students' satisfaction. Quality in higher education, 17(2), 231-243. 